\title{
Environmental Factors Regulate the hlyE Gene Expression in Both S. typhi and E. coli in a Similar Way to Display Haemolytic Activity
}

\author{
Shamma $\mathrm{F}^{1}$, Ahsan $\mathrm{N}^{1}$, Islam $\mathrm{MJ}^{1}$, Ahsan $\mathrm{CR}^{2}$ \\ ${ }^{1}$ Department of Genetic Engineering and Biotechnology, University of Dhaka, Dhaka, Bangladesh \\ ${ }^{2}$ Department of Microbiology, University of Dhaka, Dhaka, Bangladesh
}

e-mail: crahsan@du.ac.bd

\begin{abstract}
Haemolysin (HlyE) is an essential virulence factor of Salmonella, Escherichia coli and other enteric bacteria. Although, a substantial degree of haemolytic activity is not seen under normal culture conditions in these organisms, however, the non-haemolytic E. coli K-12 showed significant haemolytic activity under stress conditions. To confirm this phenomenon in other enteric bacteria, in this study, the production of haemolysin in Salmonella enterica serovar Typhi under stress conditions, like oxygen and glucose starvations in vitro was investigated during March-December 2015. For this, S. typhi was cultured under oxygen or glucose starvation condition separately and this organism showed high haemolytic activity. The activity was found to be much higher when both the conditions were applied together. Also, the role of the transcription factor SlyA of $S$. typhi was investigated on induction of haemolytic activity. When E. coli K-12 was transformed with plasmid containing the gene of SlyA, the recombinant bacteria without any starvation condition, also showed similar haemolytic activity that was exhibited by $S$. typhi grown under oxygen and glucose starvation conditions. All these findings suggest that both environmental factors like oxygen or glucose starvation and overexpression of the transcription factor SlyA have important role in inducing hlyE gene expression in S. typhi.
\end{abstract}

Key words: Glucose starvation; Haemolytic activity; hlyE gene; Oxygen starvation; Salmonella typhi

\section{Introduction}

Salmonellae are the causative agents of a variety of diseases varying from local infections of the intestinal tract to systemic forms like typhoid fever. ${ }^{1}$ Typhoid fever is a fatal illness caused by Salmonella enterica serovar Typhi (S. typhi). Worldwide, about 21.7 million cases of illnesses and 2,17,000 cases of deaths caused by typhoid fever are reported annually. ${ }^{2}$ South-central Asia and South-east Asia are the regions with high prevalence of typhoid fever (>100/100,000 cases/year). ${ }^{3}$ The disease is much more severe in Bangladesh, especially in young children where the $S$. typhi infection is substantially higher. ${ }^{4}$ The proteomic analysis showed that the crucial steps of pathogenesis of S. typhi are a result of the production of a particular toxin, the haemolysin, a product of $h l y E$ gene..$^{5}$ Like many other poreforming toxins, the HlyE toxin is also an important virulence factor among bacteria belonging to the Enterobacteriaceae. ${ }^{6,7}$ The HlyE, also denoted as ClyA and SheA, belonging to the family cytolysins, forms large, stable pores in target membranes. ${ }^{8}$ This toxin also causes haemolysis of erythrocytes and has apoptogenic effects on human and murine monocytes/ macrophages. ${ }^{9,10}$ It has been reported that genes coding for close homologues of haemolysin are present in the $S$. enterica serovar Typhi or serovar Paratyphi A and Shigella flexneri genomes. This haemolysin is also required for survival of the bacteria within the host macrophage. ${ }^{11,12}$ Furthermore, the wild-type S. typhi and S. paratyphi A strains contain functional HlyE proteins, suggesting that the HlyE protein plays important roles in the pathogenesis of these organisms. ${ }^{8}$ Interestingly, the hlyE gene encoding this potentially toxin 
protein haemolysin is also present in the nonpathogenic Escherichia coli K-12 strain, which, however, normally exhibits no haemolytic activity under standard culture conditions. ${ }^{13}$ This could be due to the fact that several other conditions may influence the regulation of $h l y E$ gene expression in E. coli $\mathrm{K}-12 .{ }^{12}$ In addition to the above findings, the non-haemolytic E. coli $\mathrm{K}-12$, on the other hand, showed significant haemolytic activity under stress conditions like oxygen and glucose starvation, indicating that stress conditions confer haemolytic phenotype properties upon the non-haemolytic $E$. coli K$12 .{ }^{14}$ In the same study, an underlying molecular mechanism responsible for inducing hlyE gene expression in response to glucose starvation and anaerobic conditions in E. coli $\mathrm{K}-12$ was also investigated by genetic analyses. Again, the $h l y E$ gene has also been found to be present in S. typhi. ${ }^{15}$ All the previous findings encouraged the authors to confirm if the "stress" phenomenon is also applicable to other enteric bacteria like S. typhi. Therefore, in this study, the haemolytic activity of $S$. typhi under environmental stress conditions, like glucose and oxygen starvation, separately and together was investigated. Furthermore, conditions other than stress that induced haemolytic activity were investigated and it was found that recombinant $E$. coli, expressing the gene for transcription factor SlyA (Salmolysin) exhibited high haemolytic activity when cultured in normal conditions without any stresses.

\section{Materials and Methods}

Bacterial strains: All the organisms used in this study were obtained from the stock culture of the Department of Microbiology, University of Dhaka. The study was conducted during the period of March-December 2015.

Animal: New Zealand white rabbits $(2-2.5 \mathrm{Kg}$ body weight) were maintained in the Department of Microbiology, University of Dhaka and all experiments using animals were undertaken following ethical issues set by the Faculty of Biological Sciences, University of Dhaka.

Homology analysis of haemolysin of S. typhi and E. coli: Haemolysin sequence of $S$. typhi (NP_805266.1) and E. coli (AP_001807.1) were obtained from the protein sequence database Genpept (www.ncbi.nlm.nih.gov) and the homology between their amino acid sequences was determined using bioinformatics software tool ClustalW2.

Homology analysis of crp and fur genes of $S$. typhi and E. coli: Sequences of the genes crp [NC_003198.1 (4213325..4213957)] and fnr [NC_003198.1 (1355387..1356181)] of S. typhi and the sequences of the genes crp [NC_000913.3 (3486120..3486752)] and fnr [NC_000913.3 (1398774..1399526)] of E. coli were obtained from the Gene sequence database (www.ncbi.nlm.nih.gov). The homology analyses of the genes were performed using the bioinformatics software BLASTn.

Culturing S. typhi and E. coli under different conditions: To observe the haemolytic activity, the $S$. typhi or E. coli K-12 strains were grown in both normal condition or under different stress conditions. For normal culture condition, organisms were grown in Luria broth containing $0.2 \%$ glucose and incubated aerobically for 24 hours at $37^{\circ} \mathrm{C}$. The stress conditions were applied by oxygen or glucose starvation, either separately or together. For oxygen starvation condition, both organisms were grown in Luria broth with glucose supplement $(0.2 \%)$ in sealed bottles and incubated in an anaerobic condition. On the other hand, for the glucose starvation condition, both organisms were grown aerobically but without any glucose supplement. Again, for both glucose and oxygen starvation conditions, both of these organisms were cultured without any glucose supplement in sealed bottles and incubated anaerobically. All experiments were repeated twice to confirm the reproducibility of the results.

Protein extraction from bacteria: After $20 \mathrm{~h}$ of incubation of S. typhi or E. coli K-12 at normal or under different stress conditions, the antibiotic Polymyxin B was added to the media $(5 \mu \mathrm{g} / \mathrm{ml})$ and cultures were further incubated for four more hours to break down the cells. Bacterial cultures were then centrifuged at $6,000 \mathrm{rpm}$ for $10 \mathrm{~min}$ and the supernatants containing the bacterial proteins were collected.

Assay of haemolytic activity: The supernatants containing the bacterial proteins were analyzed for haemolytic activity, ${ }^{13}$ where $0.5 \mathrm{ml}$ of $1 \%$ rabbit red blood cell (RBC) solution was mixed with $4.5 \mathrm{ml}$ of each of the bacterial proteins extracted from either $S$. typhi or E. coli K-12. 
The tubes were then incubated for two hours at $37^{\circ} \mathrm{C}$ followed by centrifugation at $1,500 \mathrm{rpm}$ for $10 \mathrm{~min}$ to pellet the erythrocytes. Absorbances at $543 \mathrm{~nm}$ were recorded spectrophotometrically to measure the amount of hemoglobin released into the supernatants.

Cloning of slyA gene: Isolation of genomic DNA, plasmid DNA and all DNA cloning procedures were carried out following the methods described by Sambrook et al. ${ }^{16}$ The gene slyA was amplified by Polymerase Chain Reaction (PCR) (Forward primer 5'-GCGTCAGACATGCATGC TTTAG-3'; Reverse primer 5'-GGTTACTGTCT GTCGACGCTAAACC-3'). ${ }^{8}$ After restriction digestion of both purified PCR product and pACYC184 vector $\left(\mathrm{Cm}^{\mathrm{r}}\right)$ with $S p h \mathrm{I}$ and SalI, the insert was cloned into the dephosphorylated purified vector by cohesive end ligation reaction using DNA Blunting and Ligation Kit (K1512, Fermentas, UK). The successful ligation of slyA to the vector (pACYC184-slyA vector-insert construct) was confirmed by transformation into the chemo-competent $E$. coli DH5a cells. Molecular size of the vector-insert constructs isolated from the E. coli DH5 $\alpha$ was confirmed by gel electrophoresis. Upon confirmation, pACYC184-slyA vector-insert constructs were introduced into the chemo-competent $E$. coli K12 cells and the transformed colonies were selected on chloramphenicol $(12.5 \mu \mathrm{g} / \mathrm{ml})$ containing plates.

Statistical analysis: All data were statistically analysed using 'Student's t-distribution' to compare the differences in haemolytic activities of the extracted proteins at different culture and stress conditions.

\section{Results}

Haemolytic activities of cell extracts of S. typhi grown under stress conditions like oxygen or glucose starvation, either separately or together, were found to be significantly higher when compared with the normal growth conditions (figure 1). Interestingly, the haemolytic activity was found to be higher in glucose starvation than in oxygen starvation $(p<0.001)$. Again, the haemolytic activity exhibited by $S$. typhi was found to be the highest when both of these starvation conditions were applied together than any of the stresses applied alone $(p<0.001)$.

The study also showed that the E. coli $\mathrm{K}-12$ grown under oxygen and glucose starvation conditions, either alone or together, had similar pattern of haemolytic activities like that of $S$. typhi grown under the same conditions (figure II). This result also indicates that, under separate oxygen or glucose starvation conditions, S. typhi showed 1.51 folds and 1.11 folds more haemolytic activities, respectively, when compared with the haemolytic activities of $E$. coli K-12 under same conditions. Again, the magnitude of hemolysis was found to be highest in E. coli $\mathrm{K}-12$, when both oxygen and glucose starvation were applied togther, and the value was comparable to that of the haemolytic activity shown by $S$. typhi grown under the same conditions (figure II). However, the haemolytic activity displayed by $E$. coli under oxygen starvation condition was $51 \%$ higher and under glucose starvation condition was $11.8 \%$ higher than that displayed by $S$. typhi under the same conditions.

In addition to the stress factors, other conditions that might induce haemolytic activity in S. typhi were also investigated. As previously reported, the SlyA, a transcription factor that positively regulates haemolysin production, is present in both $S$. typhi and E. coli K-12.13,15 To assess the effect of over expression of SlyA on induction of haemolytic activity in normal condition, i.e. without any stress, the slyA gene from $S$. typhi was isolated and cloned into $E$. coli $\mathrm{K}-12$. The transformed $E$. coli was then grown under normal condition and the degree of haemolytic activity was observed. When the cell extract of transformed cells was observed for the haemolytic activity, surprisingly a significant degree of haemolysis of RBC was found to occur, when compared with the cell extract of the non-transformed E. coli K-12 (figure 3) 


\section{Discussion}

The higher haemolytic activities showed by $S$. typhi at glucose and oxygen starvation conditions (figure 1).

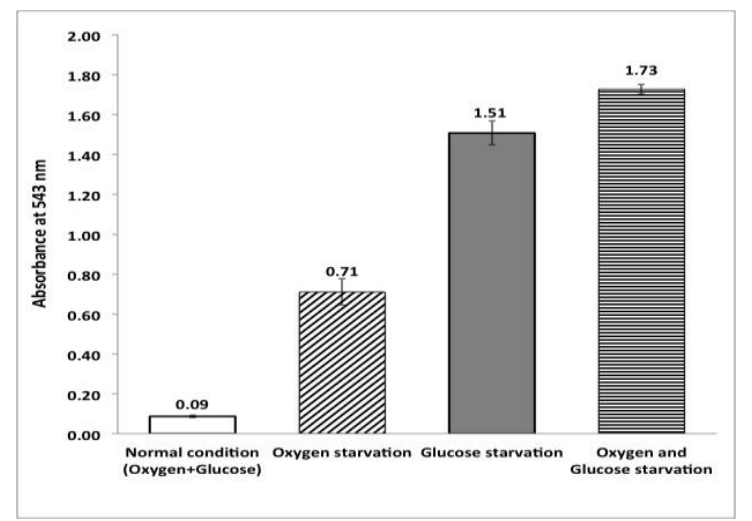

Figure 1: Haemolytic activities of cell extracts of S. typhi grown under normal and stress conditions like oxygen or glucose starvation, either separately or together. Haemolytic activities of the $S$. typhi grown under stress conditions were found to be significantly higher when compared with the normal condition $(p<0.001)$.

indicated that these particular stresses may be important for turning on the hlyE gene responsible for hemolysin production in S. typhi that ultimately caused hemolysis. This result was in accordance with the findings of Westermerk et $\mathrm{al}^{14}$ where the non-haemolytic E. coli K-12 also showed significant haemolytic activity under stress conditions.

Again, the comparison of the haemolytic activities of S. typhi and E. coli (figure 2)

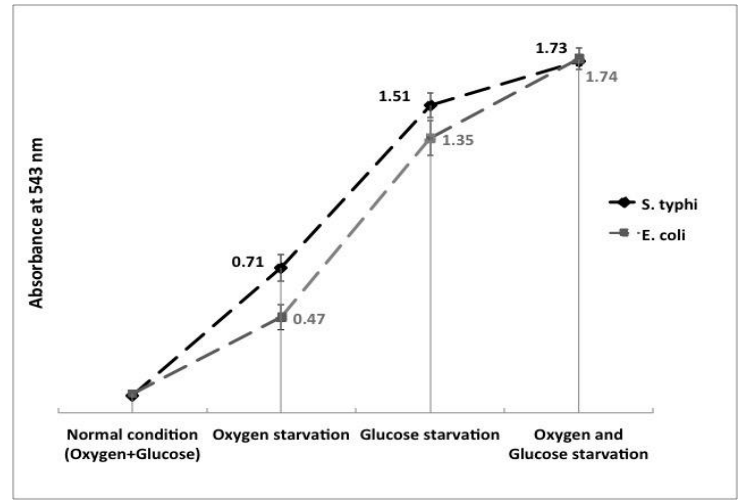

Figure 2: Comparison of haemolytic activities of cell extracts of $S$. typhi with that of E. coli K-12 grown under normal and stress conditions like oxygen or glucose starvation, either separately or together. Under separate oxygen or glucose starvation conditions, S. typhi showed more haemolytic activities when compared with the haemolytic activities of $E$. coli $\mathrm{K}-12$ under same conditions $(p<0.001)$.

clearly indicated that the haemolytic activities induced in $S$. typhi under stress conditions is quite consistent to our predictions, since $S$. typhi also harbors $h l y E$ gene like that of $E$. coli K-12. Though the hlyE gene is present in E. coli $\mathrm{K}-12$, however, it is silent in normal culture condition and its expression can be induced through stress conditions. It has been reported that two members of the cAMP Receptor Protein (CRP) family of transcription factors control the expression of $h l y E$ in $E$. coli $\mathrm{K}-12$, where CRP enhances $h l y E$ expression in response to glucose starvation and Fumarate Nitrate Reduction (FNR) regulatory protein enhances hlyE expression in response to oxygen starvation. ${ }^{15}$

In this context, using bioinformatics analysis, in this study it was observed that the genes $c r p$ and fnr of transcription factors CRP and FNR respectively, were both found in $S$. typhi and these two genes of $S$. typhi were $88 \%$ identical with the crp and fnr genes of E. coli K-12, respectively. It was also observed that both $S$. typhi and E. coli $\mathrm{K}-12$ contain functional homologs of $h l y E$ and the protein encoded by the $S$. typhi is $90 \%$ identical in amino acid sequence to that of the hlyE of E. coli K-12. In reality, the $S$. typhi does not express this gene in normal culture conditions like that of E. coli K-12. Therefore, it is evident from the current study that $S$. typhi has similar strict control mechanisms for the $h l y E$ gene and expression of $h l y E$ gene can be induced in $S$. typhi under oxygen and/or glucose starvation conditions that confer the haemolytic ability upon S. typhi. This novel finding clearly indicates that environmen-tal starvation conditions may act as stress factors for the induction of the $h l y E$ gene to produce hemolysin in S. typhi.

Also, the increased haemolytic activity of $E$. coli K-12 transformed with the transcription factor 
SlyA gene from S. typhi (figure 3). clearly indicated that overproduction of SlyA in E. coli $\mathrm{K}-12$ is independent of any culture condition. The result also suggests that the haemolytic activity can be induced in a different bacterial strain when the gene for transcription factor SlyA was cloned from another bacterial strain. This finding supports the phenomenon that SlyA overproduction antagonizes the negative effects of the regulatory protein $\mathrm{H}-\mathrm{NS}$, a nucleoid structuring protein that represses $h l y E$ gene expression. ${ }^{15}$

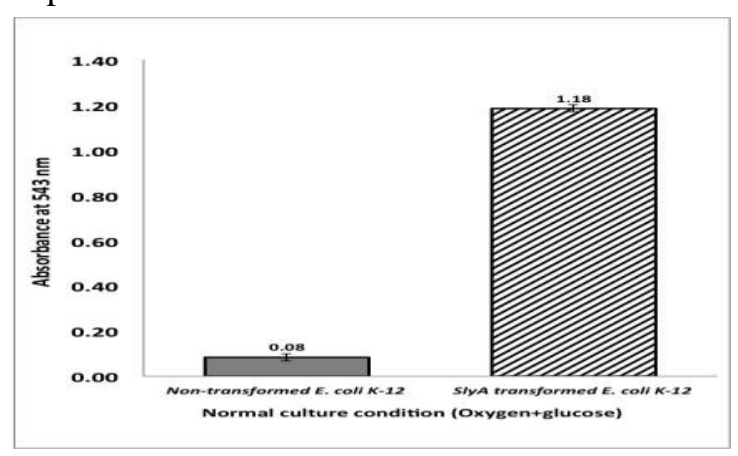

Figure 3: Comparison of haemolytic activities of cell extracts of non-transformed E. coli $\mathrm{K}-12$ and slyA gene transformed $E$. coli $\mathrm{K}-12$ grown under normal culture condition (Oxygen+Glucose). SlyA gene transformed $E$. coli $\mathrm{K}-12$ showed higher degree of haemolytic activity compared to the haemolytic activity shown by the nontransformed E. coli K-12 ( $p<0.001)$.

Based on this findings of molecular mechanisms, we assessed the degree of haemolysis seen in the haemolytic assay with RBC, after overexpression of slyA gene under normal culture conditions, and all results indicated that $h l y E$ gene might be expressed in response to appropriate environmental signals.

Haemolytic activity can be considered to be one kind of virulence factor. ${ }^{17}$ Therefore, findings of this study are very significant in clinical perspectives, since stress conditions may induce virulent properties in bacterial strains. There is evidence that stresses like starvation, acidic $\mathrm{pH}$ and heat shock may induce the expression of some virulence genes. ${ }^{18}$ Again, it has been suggested that conditions stimulating the production of the haemolysin might be encountered during infection by $E$. coli strains. $^{13}$ As both $S$. typhi and E. coli are enteric bacteria, therefore, it is assumed that these organisms may encounter oxygen starvation condition in intestinal environment which may induce the production of haemolysin in the host. This is again supported by the report that anaerobiosis has been shown to induce the invasion phenotype in Salmonella. ${ }^{19-21}$ Hence, during the course of their infection, these bacteria may encounter anaerobic condition or limited glucose, which may induce haemolysin production. Therefore, all our results along with reports of other investigators clearly indicate that, $S$. typhi may exhibit haemolytic activity under glucose or/and oxygen starvation conditions. Again, the SlyA transcription factor may also induce haemolysin production, if it is overexpressed in vivo, without going through any starvation conditions.

\section{Conclusion}

Findings of this study may also help to understand how Salmonella survive in the macrophages and how the haemolysin may help $S$. typhi in causing infections as a crucial virulence factor. Therefore, the haemolysin protein can be a potential antigenic target for development of a vaccine as an alternative therapeutic agent for Salmonella infections. Previously, in Bangladesh, studies on anti-HlyE responses in patients have been carried out with a view to developing improved diagnostic assays. ${ }^{22}$ In this novel study, it was assayed production of HlyE toxin under various conditions in order to analyse this protein with a view to developing a novel vaccine against this toxin in future in Bangladesh.

Acknowledgments: This project has been partially funded by the University Grants Commission (UGC) Bangladesh. Author would like to heartily thank Dr. Siraje Arif Mahmud for his expert assistance with this manuscript.

\section{References}

1 Ludwig A, Tengel C, Bauer S, Bubert A, Benz R, Mollenkopf HJ, et al. SlyA, a regulatory protein from Salmonella typhimurium, induces a haemolytic and pore-forming protein in Escherichia coli. Mol Gen Genet. 1995; 249:474-86.

2 Crump JA, Mintz ED. Global trends in typhoid and paratyphoid Fever. Clin Infect Dis. 2010; 50(2):241-6.

3 Crump JA, Luby SP, Mintz ED. The global burden of typhoid fever. Bulletin of World Health Organization. 2004; 82:346-53. 
4 Saha SK, Baqui AH, Hanif M, Darmstadt GL, Ruhulamin M, Nagatake T, et al. Typhoid fever in Bangladesh: Implications for vaccination policy. Pediatr Infect Dis J. 2001; 20 (5):521-24.

5 Ansong C, Yoon $\mathrm{H}$, Norbeck AD, Gustin JK, McDermott JE, Mottaz HM, et al. Proteomics analysis of the causative agent of typhoid fever. J Proteome Res. 2008; 7:546-57.

6 Alouf JE. Pore-forming bacterial protein toxins: an overview. Curr Top Microbiol Immunol. 2001; 257:1-14

7 Parker MW, Feil SC. Pore-forming protein toxins: from structure to function. Prog Biophys Mol Biol. 2005; 88:91-142.

8 vonRhein C, Hunfeld KP, Ludwig A. Serologic evidence for effective production of cytolysin $\mathrm{A}$ in Salmonella enterica serovars Typhi and Paratyphi A during human infection. Infect Immun. 2006; 74:6505-08.

9 Lai XH, Arencibia I, Johansson A, Wai SN, Oscarsson J, Kalfas S, et al. Cytocidal and apoptotic effects of the ClyA protein from Escherichia coli on primary and cultured monocytes and macrophages. Infect Immun. 2000; 68:4363-67.

10 Oscarsson J, Westermark M, Lofdahl S, Olsen B, Palmgren $\mathrm{H}$, Mizunoe $\mathrm{Y}$, et al. Characterization of a pore-forming cytotoxin expressed by Salmonella enterica serovars Typhi and Paratyphi A. Infect Immun. 2002; 70 (10):5759-69.

11 Libby SJ, Goebel W, Ludwig A, Buchmeier N, Bowel F, Fang FC, et al. A cytolysin encoded by Salmonella is required for survival within macrophages. Proc Natl Acad Sci. 1994; 91:489-93.

12 Hunt S, Green J, Artymiuk PJ. Hemolysin E (HlyE, ClyA, SheA) and related toxins. In: Proteins: Membrane Binding and Pore Formation. V 677. Anderluh G, Lakey J editorss. New York, USA, Springer Science+Business Media, 2010, p 116-26.

13 Ludwig A, Bauer S, Benz R, Bergmann B, Goebel W. Analysis of the SlyA-controlled expression, sub- cellular localization and pore-forming activity of a $34 \mathrm{kDa}$ haemolysin (ClyA) from Escherichia coli K12. Mol Microbiol. 1999; 31:557-67.

14 Westermark M, Oscarsson J, Mizunoe Y, Urbonaviciene J, Uhlin BE. Silencing and activation of ClyA cytotoxin expression in Escherichia coli. J Bacteriol. 2000; 182(22):6347-57.

15 Wyborn NR, Stapleton MR, Norte VA, Roberts RE, Grafton J, Green J. Regulation of Escherichia coli Hemolysin E expression by H-NS and Salmonella SlyA. J Bacteriol. 2004; 186 (6) :1620-28.

16 Sambrook J, Fritsch EF, Maniatis T. Molecular Cloning: a Laboratory Manual: 2nd ed. New York: Cold Spring Harbor Laboratory Press; 1989.

17 Goebel W, Chakraborty T, Kreft J. Bacterial hemolysins as virulence factors. Antonie van Leeuwenhoek. 1988; 54:453-63.

18 Mekalanos JJ. Environmental signals controlling expression of virulence determinants in bacteria. $\mathbf{J}$ Bacteriol. 1992; 174 (1):1-7.

19 Ernst RK, Dombroski DM, Merrick JM. Anaerobiosis, type 1 fimbriae, and growth phase are factors that affect invasion of HEp-2 cells by Salmonella typhimurium. Infect Immun. 1990; 58:2014-16.

20 Lee CA, Falkow S. The ability of Salmonella to enter mammalian cells is affected by bacterial growth state. Proc Natl Acad Sci. 1990; 87:4304-08.

21 Schiemann DA, Shope SR. Anaerobic growth of Salmonella typhimurium results in increased uptake by Henle 407 epithelial and mouse peritoneal cells in vitro and repression of a major outer membrane protein. Infect Immun. 1991; 59:437-40.

22 Charles RC, Sheikh A, Krastins B, Harris JB, Bhuiyan MS, LaRocque RC, et al. Characterization of anti-Salmonella enterica serotype Typhi antibody responses in bacteremic Bangladeshi patients by an immunoaffinity proteomics-based technology. Clin Vaccine Immunol. 2010; 17(8):1188-95. 